\title{
Oil Spill Response System using Server-client GIS
}

\author{
†Hye-jin Kim, Moonjin Lee*, Se-woong Oh** \\ + Marine Safety \& Pollution Response Research Department, KORDI, Daejeon, 305-343, Republic. of Korea \\ (Division of Civil Engineering, Chungnam National Univ. Daejoen, 305-764, Republic. of Korea) \\ *, ** Marine Safety \& Pollution Response Research Department, KORDI, Daejeon, 305-343, Republic. of Korea
}

\begin{abstract}
It is necessary to develop the one stop system in order to protect our marine environment rapidly from oil spill accident. The purpose of this study is to develop real time database for oil spill prediction modeling and implement real time prediction modelling with ESI and server-client GIS based user interface. The existing oil spill prediction model cannot provide one stop information system for public and government who should protect sea from oil spill accident. The development of multi user based information system permits integrated handling of real time meteorological data from external ftp. A server-client GIS based model is integrated on the basis of real time database and ESI map to provide the result of the oil spill prediction model. End users can access through the client interface and request analysis such as oil spill prediction and GIS functions on the network as their own purpose.
\end{abstract}

Key words : oil spill, ESI map, real time data, one stop system, server-client GIS

\section{Introduction}

Oil spill accidents in the marine environment can strike with no warning and it may give rise to serious problems in various aspects. Most of problems related to oil spills have greatly continued over long time since oil spill accident occurred such like Hebei Sprit Oil Spill Accident (2007.12. 7). Because of a lot of the various human activities in the sea area, the damage caused by oil spill is very serious. An effective real-time oil trajectory modelling and GIS information system can help mitigate the worst effects of oil spills through the rapid dissemination of prediction information regarding threatened area. If maps of potential oil spill diffusion and risk analysis considering ESI(Environment Sensitivity Index) are provided, it will be easy to decide the appropriate response strategy and share the potential risk information with public.

In this study, the oil spill response system for protect marine environment has announced and components of the system have described. This system is the first one stop information system in order to produce the scientific information easily and rapidly through one single interface one the network. We have two goals in this paper:

1. to describe requirements of the oil spill response information system and

2. to introduce the development of a new one stop information system for interactive operation

\section{Requirements of GIS based system}

\subsection{Real-time data}

Real-time data refers to spatial and non-spatial data that becomes available to the real-time GIS, either at fixed time intervals or after the completion of certain events such as the arrival of data at a desired destination. Conventional GIS models such as data modeling, data management, and software design and engineering do not allow current GIS systems to meet the requirements of real-time applications effectively (Karimi \& Chapman, 1997). The key requirement of real-time oil spill prediction for the appropriate response, however, is based on continuous in meteorological forecasting to improve the accuracy of prediction model. While there have been significant advances in the accuracy of weather forecasting through the super computer, extensive research efforts are still required to develop systems that incorporate real-time data with a GIS application so that real-time data can be obtained with sufficient timeliness and rapid nowcasting can be produced. Interfacing of GIS with external systems for real-time processing is difficult. Real-time GISs must contain algorithms that allow fast responses within time constraints for real-time application. Because many GIS algorithms do

\footnotetext{
† Corresponding Author, hjk@moeri.re.kr 042)866-3649

* Moonjin.Lee@moeri.re.kr 042)866-3614

** osw@moeri.re.kr 042)866-3692
} 
not take these constraints into consideration, they cannot be used for real-time applications.

In this study, there are three basic data system for providing meteorological data that can be used for real-time sea meteorological prediction: First, air temperature data is forecasted by Korea Meteorological Agency, which is bi-daily provided through KMA ftp server. A second wind forecasting data is from KMA, which is also bi-daily provided through KMA ftp server. Third data is the sea surface temperature from NOAA satellite image which is daily provided through ftp server of Tohoku university. These data acquisition systems are interfaced to different ftp servers and these datasets have different spatial coverage and different resolution. So data extracting system is required. After collecting datasets from different ftp server, the datasets with grid coverage and cell size are generated by the data extracting system. For a GIS to be effectively applied in the information system, up-to-date sources are required. Although, weather forecasting systems exist and deployed in many projects, constant utilization of real-time weather forecasting data is rare in real time based information system.

\subsection{ESI (Environmental Sensitivity Index) map}

There are various sensitive resources to oil pollution such as fishery place, farms, beach, and biological habitats at coastal area. The methods of oil spill response depend mostly on geographical features of these sensitive resources. In order to cope with an accident efficiently, the response strategy should be decided according to the geographical features of sensitive resources and the mobilization ability of the response resources. ESI map is a good guide to make the most optimum decision. ESI maps include a lot of information about the sensitivities of shoreline, biology and socio-economy.

Shoreline features are most important information to make a coastal response strategy. Shoreline features about the oil accidents are classified the characteristics of shore slope, geology, wave, ebb and flow which are have an effect on removal, adherence and saturation of oils. The sensitivity classification of the shoreline had been studied for a long time but that classifications cannot be general standards because of the geographical diversity. Korean shoreline indexes have been defined with reflecting Korean coastal topography such like large tidal mud flats and rias shorelines(Kim, et al. 2006). Shoreline and coastal geomorphologic information are extracted from Korean digital maps, nautical charts (1:25,000) and satellite images.
Sometimes field surveys were carried out.

There are diverse species in Korean coastal area and some of them are sensitive to oil spills. First, kinds of sensitive species are defined, which are salt plants, mammals, birds, fishes, mollusks, crustacean and et al. Biological system is very complex as we know that the classification divides into species, genera, families, orders, classes and so on. It is difficult to classify the sensitivity index of species like shoreline. Accordingly, biological datasets are built by biology professionals' advices of each area. This information consists of habitats, breeding places, appearance seasons and so on.

There are various human activities and dense facilities in Korean coastal area and then diverse items about socio-economic features should be built. These datasets are divided coastal resource utilities, water front places, port facilities and national protected area and each items have some sub items. This broad information is provided from local governments, national park offices and port authorities.

Not only geographic sensitivity information but also response resource information is very important to decide a response strategy. Mobilization capability of response equipments influences on a prompt response. Response resources are distributed at Korea coast guards, port administrations, response companies and KOEM(Korea Maritime Environment Management Corporation) branches. They are categorized by response ships, response equipments and response materials. Items of response ships are classified with workboats, oil tankers, tug boats, waste oil tankers, oil fence building ships and others. Their owner, ship size, weight, maximum speed, communication methods, oil storage volume, oil recovery and storage methods, and included equipments are provided by GIS attributes. Response equipments and materials are including oil fences, oil adsorbents, dispersant, storage tools, oil cleaners, spray tools, high pressure cleaners and so on. Korean ESI map provides the locations, organizations, contact points and stockpiles of response resources as GIS database (location and attribute are combined).

\subsection{User's operation environment}

During the development of a system, the most significant aspect of implementation is an appropriate user interface, for it determines the interaction between the computer system and the user (Dodson, 1993). This interface permits the application of a variety of tools regulating both the visualization and analysis of the spatial distribution of model parameters as well as the simulated variables set as a 
variety of spatial and temporal scales. The quality of the user interface connecting the GIS has become an essential contributing factor motivating people to use GIS as a means of handling spatial data and for making decision. The user interface is the most important single part of the system(Frank, 1993). Albercht et al. (1997) stated that the finished product of a modeling system must include a graphical user interface that is simple to use. Knill (1993) stresses the need for improvements in user-friendliness, including visualization and results.

GIS and simulation models are used most frequently in professional and academic endeavors. Therefore, the development in oil spill prediction model integration with GISs is actually the result of the development of interfaces that facilitate the creation of oil spill model input data sets. Models such as GNOME which lack good interface design require intensive data development for input which is often very labour intensive and time consuming. Because interfacing is the most important part of oil spill response system development, the need for improvement in user-friendliness, and flexibility is definitely needed in order to facilitate the use of such system.

Environmental information is usually held in government and academic. Oil spill modeling tends to remain in the domain of the model developer and to be applied within a consulting framework. The models are inaccessible to oil spill response decision makers who are not modeling specialist and appear to be designed for experts and professionals for use as in-house tools. While data are currently available for public use through the network by some institutions and governmental agencies, many of these institutions, however, provide the data in varying formats and use complicated relational database. Unfortunately, effective use of these data requires specialized software not available to every user.

It has long been recognized that GISs provided the user with a flexible framework for the development of GIS-based SDSSs(Spatial Decision Support Systems) (Clarke, 1990). A GIS is often designed for spatial decision support, lacking the capacity for collaborative spatial decision-making (Jankowski, Nyerges, Smith, Moore \& Horvath, 1997). However, in the future, a GIS must support SDSSs in collaborative environments (Karimi \& Blais, 1997) designed for sharing, executing, and comparing model results, especially when the decision makers and/or stakeholders are from geographically separate locations (Carver Frysinger, Reitsma, 1996).

In this study, real-time data acquisition system, ESI map layers and GIS based user interface have developed and integrated into one system using the one network. According as sever-client based one system has operated, multi user accessibility has set aside.

\section{System implementation}

\subsection{Numeric model}

Despite advances in computer and programming languages, FORTRAN remains the language of mathematical modelers. In this study, calculation model has implemented by FORTRAN and other parts such as map utilization and server-client programming have implemented by $\mathrm{C}++$.

We have used the oil spill prediction model(Kim and Lee, 2008) that provides a quantitative description and understanding of physical processes. The main purpose of prediction modelling unit is to process the incoming data by applying physical analysis and then to produce the desired results. The results of this numeric model are non-spatial (time series) and spatial (grid) of different variables (SST, current, etc).

For the real time tidal current modeling, CHARRY (Currents by Harmonic Response to the Reference Yardstick, Kang, 1999) model has used. Wind driven surface current is directly calculated from the real time wind data from KMA and its distribution was derived from the result of Lee and Kang (2000) which had suggested relation between the wind and the sea surface wind-induced drift.

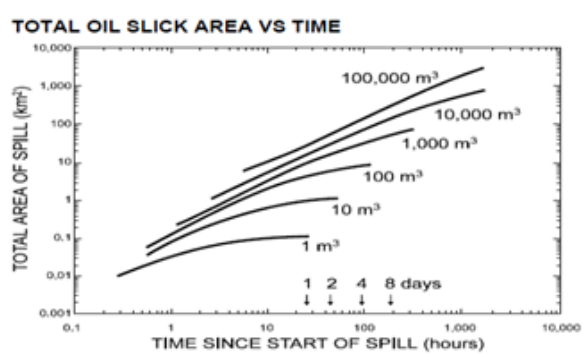

Fig. 1 Oil slick area with time

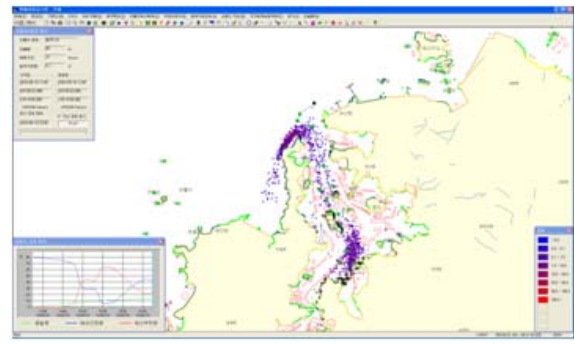

Fig. 2 Result of oil spill prediction 
Spilled oil drifts and disperses with the current and turbulent flow and quantities of floating oil decrease by evaporation and dispersion and increase by emulsification. Numerical tracer with Monte Carlo method which corresponds to the spilled oil has adopted to predict the oil spill trajectory and weathering for the spilled oil were extracted using data from National Oceanic and Atmospheric Administration (NOAA) and International Tank Owners Pollution Federation Limited (ITOPF). Numerical prediction model for the oil spill has carried out with numerical tracer method and the weathering process modeling considering the characteristics of the spilled oil.

\subsection{Map utilization}

User interface designed for map utilization has developed $\mathrm{C}++$ language with Object Oriented Programming concept. This client-side interface includes a hybrid map based display system. To load spatial images and the results obtained by applying the real time data query functions (e.g. SST, current, etc.), a rendering algorithm is developed and it extracts the real time information or the spatial function results and then displays the image on the fly. This client-side interface is designed in order to reduce network traffic and processing burden on the server when displaying large data such as ESI map. Once the ESI map is displayed, users can select from the different buttons allowing them to adjust the map view. To adjust the map view, zoom-in, zoom-out, and pan are supported. The system is also capable of performing animation, permitting users to see more than one layer at a time in the same frame, thereby allowing a view of the changes of a set of ESI layers selected by the user. Before the animation application can run, client application was designed that run server's calculation model. This application then stores each calculation result in its predefined arrays so the animation applet can process when it is called. Additionally, the applet generates a time slider for requested animation. It also allows users to obtain some analytical statics on time series graph to obtain evaporated volume, remained volume and shore-attached volume at each time. The mechanics of the access and the analytical process are available to anyone on the network who has authorization.

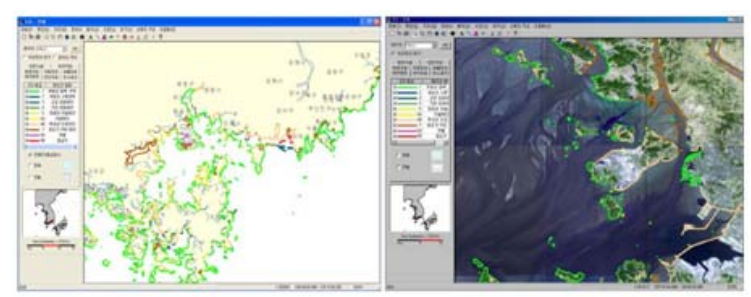

Fig. 3 ESI map display

\subsection{Server-client communication model}

The server interface has designed to store the results in specific memories. After the data have been processed by the modelling unit and the results have been stored in their specified memories, the server sends the results automatically to the client.

The server-client model can potentially contribute to the achievement of the goals of sharing oil spill analysis. The benefits and the advantages that the sever-client offers to oil spill analysis are openness, a user-friendly interface, interactivity, flexibility and fast communication. It is relatively cheap and therefore gives the general multi access to a variety of both GIS systems and data of varying degrees of sophistication. This direct access, as a means of allowing wider involvement and participation in decision-making and response actions, is an important prerequisite of response management. This accessibility occurred because of user interface familiarity, portability across many computers. Additional benefit that is offered by $\mathrm{C}++$ programming language is the development of a sophisticated user-friendly interface, which can interact with data meaningfully and efficiently. Users can easily interact with the contents of the database or run prediction models, thus creating their own analysis results. These results being spatial or non-spatial data, ocean physics, still or animated grid are processed in a server and then displayed immediately in the client machine. This fast communication via the network is probably the fastest and the most economic method. Therefore, by integrating a sever-client system with monitoring network, real-time data can be obtained and disseminated instantly. These important features and tools that enhance and improve oil spill analysis are available and therefore it becomes a question of using them efficiently and professionally.

The promising step in the development of server-client GIS systems has been the implementation of the $\mathrm{C}++$ programming language. MFC provides tools for creating graphic, networked, and database applications that are essential for building response strategy and management. $\mathrm{C}++$ features like object-orientation, GUI, platform neutrality, security, image processing, and multi-threading are useful for building such multi user applications. This system has conceived through a general need for real-time data acquisition, data management, a user-friendly interface and accessibility of the data and applications. The primary aim is to develop an integrated system that would overcome problems related to the above requirements.

This study has proposed that server-client model 
combines server function for database and model and client function for information query as following figures. In this configuration, communication between server and client has been implemented using TCP/IP protocol and server can permit multi users simultaneously.

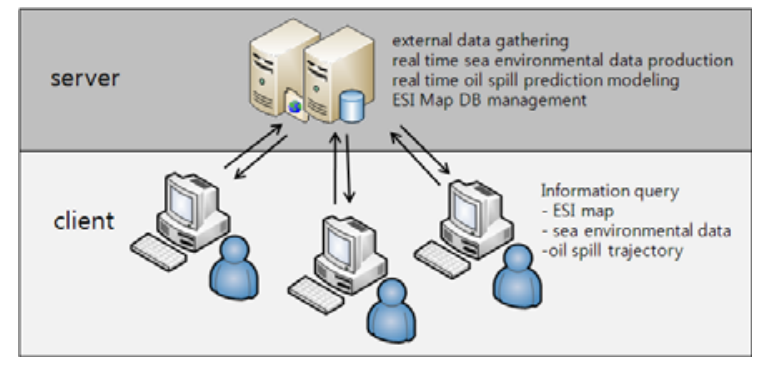

Fig. 4 Server-client configuration

\section{Conclusion}

Server-client based GIS system is necessary in order to response the accident in whole aspects including experts and public. However it is very difficult to implement one stop system because of difficulty of real time data acquisition and integration of various components. Real-time data acquisition is increasingly important in many fields, particularly in oil spill response. This study has developed practical real time data acquisition system using external database and modelling unit for oil spill modelling with real time database has combined GIS based user interface with ESI map.

This system has been operated with server-client architecture and so multi users from experts to general people can access this system. Client interface has been designed user friendly and it has various GIS functions including oil spill prediction analysis. Server interface has received the external real time data daily and then handled them to provide basic data for numerical modelling. This system is easy to manage and maintain due to modulation of system functions. And also user interface is easy to use due to GIS based application and any authorized person can utilize this system in order to make decision for responding oil spill accident.

\section{Acknowledgements}

This study is supported by the KORDI top-brand project "Development of the Support System to Response Marine Spill Accident".

\section{References}

[1] Albercht,J., Jung, S., \& Mann, S. (1997), VGIS: a GIS shell for the conceptual design of environmental models. In Z. Kemp, Innovations in GIS 4, fourth national conference on GIS research UK (GISRUK), pp. 154-165

[2] Carver, S., Frysinger, S., \& Reitsma, R. (1996), Environmental modelling and collaborative spatial decision-making: some thoughts and experiences arising from the $\mathrm{I}-17$ meeting. In Proceeding 3rd international conference and workshop on integrating geographical information systems and environmental modelling

[3] Clarke, M. (1990), Geographical information systems and model based analysis: towards effective decision support system. In H.J. Shcolten, \& J.C.H. Stillwell, Geographical information systems for urban and regional planning, pp.165-175

[4] Dodson, R.D. (1993), Advances in hydrological computation. In D. Maidment, Handbook of hydrology, pp.23.1-23.24

[5] Frank, A.U. (1993), The use of geographical information system : the user interfaces the system. In D. Medyckyj-Scott, \& H.Hearnshaw, Human factors in geographical information systems, pp. 3-14

[6] Jankowski, P., Nyerges, T., Smith, A., Moore, T.J., \& Horvath, E. (1997), Spatial group choice: a SDSS tool for collaborative spatial decision making. In P. Fisher, K.C. Clark, \& B.Lees, International journal of Geographical Information Science Vol. 11, No. 6

[7] Kang, Y. (1999), Rapid prediction of real-time currents for environmental problems, Proceedings of international symposium on progress in coastal engineering and oceanography, Seoul, Korea.

[8] Karimi, H.A.,\& Blais, J.A.R. (1997), Current and future direction in GISs. Computer, Environment and Urban Systems, Vol. 20, No. 2, pp. 85-97

[9] Karimi, H., \& Chapman, M. (1997), Real-time GISs: an emerging technology through the integration of GPS, video imagery, and fast algorithms. In proceeding of integrating spatial information technologies for tomorrow, GIS '97, pp.630-633

[10] Kim, H., Lee, H. and Lee, M., (2006), Building of GIS program for controlling oil spill accident, Journal of the Korean Association of GIS, Vol. 9, No. 3.

[11] Kim, H. \& Lee, M. (2008), Prototype development of marine information based supporting system for oil spill response, Journal of the Korean Association of GIS, 
Vol. 11, No. 4.

[12] Knill, J. (1993), Geographical information systems: the environmental view. In P. Mather, Geographical information handling-research and applications, pp.7-15

[13] Lee, M., \& Kang, Y (2000), Lagrangian observation and modeling of sea surface wind-induced drift(skin drift), Journal of Korean Society for Marine Environmental Engineering, No. 3, pp. 11-17

[14] Michel, J. and Dahlin, J.,(1993), Guidelines for Developing Digital Environmental Sensitivity Index Atlases and Database

[15] NOAA, (1995), Environmental Sensitivity Index Guideline

Received 31 October 2011

Revised 20 December 2011

Accepted 20 December 2011 